\title{
Programmable Thermal Metamaterials Based on Optomechanical Systems
}

\author{
Zhengyang Zhou, ${ }^{1,2^{*}}$ Xiangying Shen, ${ }^{3,1^{*}}$ Chenchao Fang ${ }^{3}$ and Jiping Huang ${ }^{1 *}$
}

Controlling heat flow is crucial to improving human's production efficiency and daily life. Although thermal metamaterials have played important roles in heat manipulations, as the growing demands of intelligent and multi-functional apparatuses, the requirements of more advanced metamaterials are still far from being satisfactory. Moreover, the popular thermal metamaterials such as cloak, concentrator, rotator, etc., are essentially based on particularly spatial distributions of thermal conductivity. Hence, the designs of thermal meta devices with certain functions are greatly restrained by the positive and definite conductivities of natural materials. In this article, we propose an approach to building thermal metamaterial by utilizing the optomechanical system as an elemental unit. After deriving the relationship between thermal conductivities and pump light in one such unit, we can program the value of conductivity at an arbitrary point of an array system consisting of optomechanical cells by directly adjusting the pump power. This characteristic ensures great freedoms and flexibilities in designing metamaterials and permits people achieving any effects of existing thermal devices by using only one array system.

Keywords: Optomechanical systems; Thermal metamaterial; Adjustable thermal conductivities

Received 19 September 2019, Accepted 21 October 2019

DOI: $10.30919 / \mathrm{esee} 8 \mathrm{c} 336$

\section{Introduction}

Manipulating heat flow is significant in energy saving, chips cooling, thermoelectric effects, and industrial manufacturing. Thermal metamaterials have been proved to be powerful tools in controlling heat flow at will. ${ }^{1-21}$ This sort of artificial materials are able to show some unusual behaviors that can not be found in natural materials. Nowadays, advanced applications in prosthetics, soft deformable material, and wearable technology have become more and more popular. Demands of programmable materials with intelligence ${ }^{22}$ and multifunctions are urgently needed in thermal energy storage ${ }^{23}$ and serving as building blocks of that fantastic implementations. ${ }^{24}$ However, due to the architecture and thermal conductivity are crucial to metamaterials' properties and functionalities, different types of thermal devices always have different geometrical structures and conductivity tensors. As a result, there exist large gaps between different implements. Essentially, most of the popular thermal meta devices are constructed by tailoring the spatial distribution of the devices' thermal conductivities, which is a positive and definite value for certain materials. Although we can combine different materials to fit desired conductivity under the

${ }^{\prime}$ Department of Physics, State Key Laboratory of Surface Physics, and Key Laboratory of Micro and Nano Photonic Structures (MOE), Fudan University, Shanghai 200433, China

${ }^{2}$ Theoretical Quantum Physics Laboratory, RIKEN Cluster for Pioneering Research, Wako-shi, Saitama 351-0198, Japan

${ }^{3}$ Department of Physics, Chinese University of Hong Kong, Hong Kong 999077, China

*E-mail: zhengyang.zhou@riken.jp; xiangyingshen@cuhk.edu.hk; jphuang@fudan.edu.cn instruction of effective medium approximation (EMA) theory, the upper and lower bound of the fitted conductivity are constrained to the highest and lowest conductivities of chosen materials respectively, which also restrict the freedom of designing devices. Moreover, for those thermal meta devices that emphasize noninvasively, i.e., the existence of the device makes no distortions to the temperature gradient, the effective conductivities of such metamate-rials should match with the one of background, which also narrows the application potential due to the different environments require different constituents to build the device. Therefore, an improved adjustable and pro-grammable multi-functional thermal metamaterial with a wide range of tunable thermal conductivity is of great value to be investigated.

Fortunately, several experiments have shown that the microstructures can change the thermal conductivity by manipulating the phonon modes. ${ }^{25}{ }^{26}$ Inspired by these results, we investigate the possibility of construct-ing adjustable and multi-functional metamaterial with optomechanical systems driven by a light pump. Besides the structures of the materials, the electromagnetic field can also influence the phonon modes. For example, optomechanical systems are such kind of devices that can change the phonon modes of oscillators with light ${ }^{27}$ and are usually formed by oscillating mirrors(the oscillators) coupled to light field. The amplitude (average phonon number) of the mechanical oscillators can either be increased ${ }^{28}$ or decreased ${ }^{29}$ by the light, and the rigidity of the mechanical oscillator ${ }^{30}$ is also possible to be adjusted. Thus, by changing the phonon modes, the thermal conductivity of the optomechanical systems can vary accordingly. In this article, we will first show that changing the phonon modes in optomechanical systems can be an effective way to adjust the thermal conductivity of thermal metamaterial. Then, based on optomechanical systems, we will design a new practical method to build programmable thermal metamaterials, whose functions (as mentioned before, functions are always defined by 
the thermal conductivities) can be controlled by the intensity of the pump light.

\section{Thermal conductivity of single device}

The thermal conducting unit we propose is an optome-chanical system with two mechanical modes coupled to a common optical mode. This system can be realized with an optical cavity formed by two movable mirrors as shown in Fig. 1. The displacement of the mirror will change the frequency of the cavity, which results in the coupling be-tween optical modes and mechanical modes. ${ }^{27}$ In this case, the heat flux through the device is produced by the phonon exchanges between the two mechanical oscilla-tors.

The Hamiltonian of the cavity can be expressed as

$$
H_{\mathrm{cav}}=\hbar \omega\left(x_{1}, x_{2}\right) a^{\dagger} a
$$

where $\omega$ is the frequency of the cavity mode which de-pends on the displacement of the two mechanical oscil-lators; $x_{i}, \mathrm{i}=1,2$ is the displacement of the mechanical oscillator $i ; a^{\dagger}(a)$ is the bosonic creation (annihilation) operator of the cavity optical mode. The photon number $a$ † $a$ is a conserved quantity of the Hamiltonian $H_{\text {cav }}$ in Eq. (1). So we can substitute the photon number operator $a$ † $a$ with the average photon number $n_{\mathrm{ph}}$. The photon number, which is determined by the pump intensity and the decay of the cavity, can be calculated with

$$
n_{\mathrm{ph}}=\frac{F P}{\hbar \omega \Delta \omega_{\mathrm{FSR}}},
$$

where $F$ is the finesse of the cavity, $P$ is the pump power, and $\Delta \omega_{\text {FSR }}$ is the free spectral range of the cavity. ${ }^{27}$ Then we expand the Hamiltonian in Eq. (1) to the second order of the oscillator displacement.

$$
\begin{aligned}
H_{\mathrm{cav}}= & \hbar n_{\mathrm{ph}}\left[\omega+\left(\frac{\partial}{\partial x_{1}} \omega\right) x_{1}+\left(\frac{\partial}{\partial x_{2}} \omega\right) x_{2}\right] \\
& +\hbar n_{\mathrm{ph}}\left[\left(\frac{1}{2} \frac{\partial^{2}}{\partial x_{1}^{2}} \omega\right) x_{1}^{2}+\left(\frac{1}{2} \frac{\partial^{2}}{\partial x_{2}^{2}} \omega\right) x_{2}^{2}\right] \\
& +\hbar n_{\mathrm{ph}}\left(\frac{\partial^{2}}{\partial x_{1} \partial x_{2}} \omega\right) x_{1} x_{2} .
\end{aligned}
$$

The first five terms, which change the equilibrium positions or the frequencies of the oscillators, can be absorbed into the parameters of the oscillators. Only the last term in Eq. (3) contributes to the coupling between the two mechanical oscillators. As a result, we focus on the last term, and obtain the following Hamiltonian

$$
H_{\mathrm{cav}}=\hbar n_{\mathrm{ph}}\left(\frac{\partial^{2}}{\partial x_{1} \partial x_{2}} \omega\right) x_{1} x_{2} \equiv \hbar n_{\mathrm{ph}} \omega^{(2)} x_{1} x_{2},
$$

where we define the second derivative of cavity frequency $\omega^{(2)} \equiv$ $\frac{\partial^{2}}{\partial x_{1} \partial x_{2}} \omega$. Now we quantize the mechanical oscillator. To make the case easy, we consider the single mode situation.

$$
\begin{aligned}
H & =H_{\mathrm{cav}}+H_{\mathrm{m}}, \\
H_{\mathrm{cav}} & =\hbar n_{\mathrm{ph}} \omega^{(2)} x_{\mathrm{ZPF}}^{2}\left(b_{1}^{\dagger}+b_{1}\right)\left(b_{2}^{\dagger}+b_{2}\right), \\
H_{\mathrm{m}} & =\hbar \Omega_{m} b_{1}^{\dagger} b_{1}+\hbar \Omega_{m} b_{2}^{\dagger} b_{2} .
\end{aligned}
$$

The $\Omega_{m}$ and $H_{m}$ here are the frequencies and Hamiltonian of the mechanical oscillators, respectively. $b_{i}^{\dagger}\left(b_{i}\right)$ is the bosonic creation (annihilation) operator of the phonon mode $(i=1,2)$. The $x_{\mathrm{ZPF}}$ is the zero-point fluc-tuation amplitude of the mechanical oscillator.

$$
\begin{aligned}
x_{\mathrm{ZPF}} & =\sqrt{\frac{\hbar}{2 m_{\mathrm{eff}} \Omega_{\mathrm{m}}}}, \\
x_{i} & =x_{\mathrm{ZPF}}\left(b_{i}+b_{i}^{\dagger}\right), i=1,2
\end{aligned}
$$

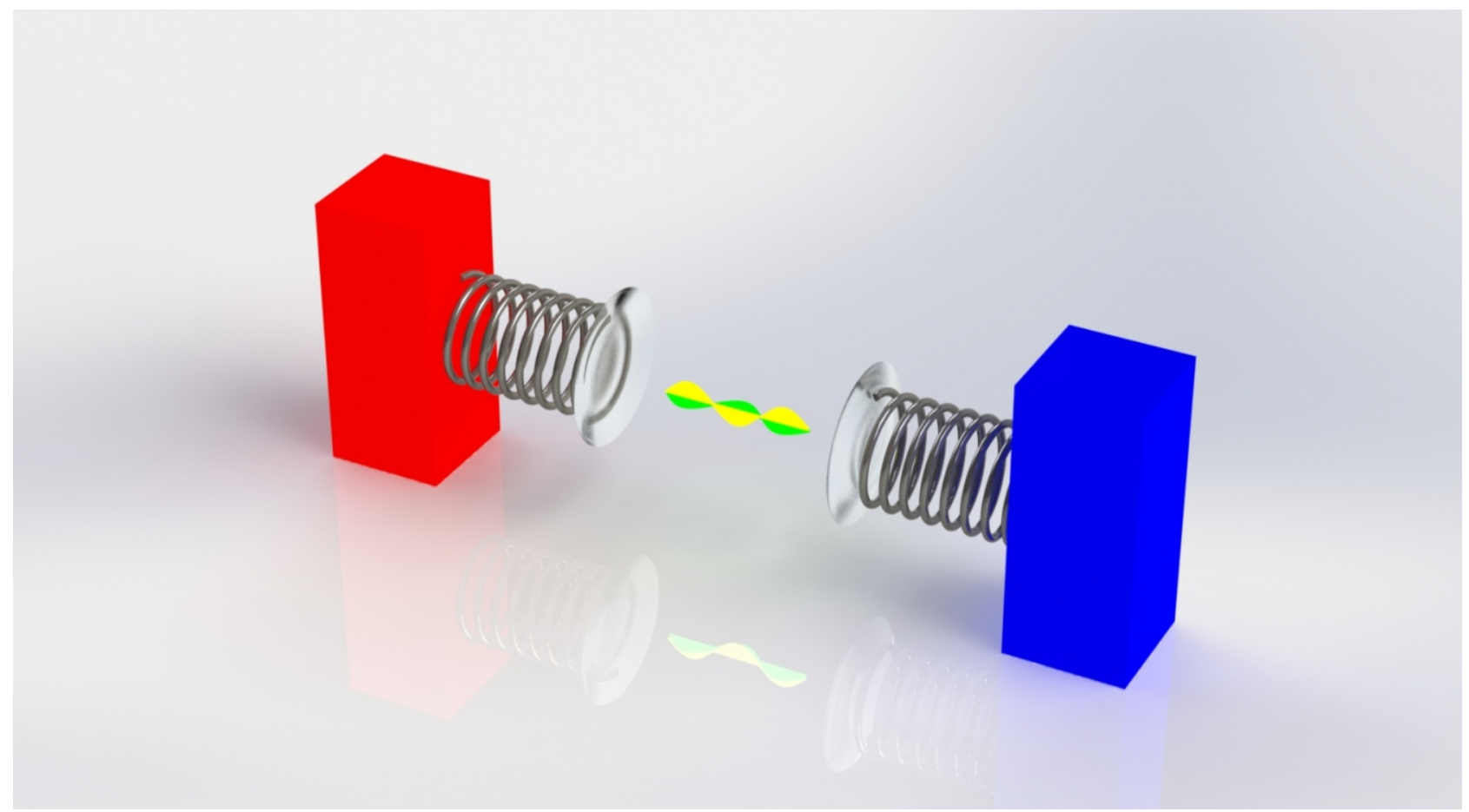

Fig. 1 The illustration of the thermal conducting device. The cavity in our scheme has two movable mirrors. These two mirrors are connected to two identical mechanical oscillators. Both mechanical modes are coupled to a thermal bath indicated by the red and blue cuboid representing the heat and cold source respectively. The intensity of the light in the cavity is controlled by the pump intensity. 
with $m_{\text {eff }}$ the effective mass of the mechanical oscillator. ${ }^{27}$ Under rotating wave approximation, the Hamiltonian of the coupling between two mechanical os-cillators in Eq. (4) becomes

$$
H_{\text {cav }}=\hbar g b_{1}^{\dagger} b_{2}+\hbar g b_{2}^{\dagger} b_{1} \text {. }
$$

where we have defined the coupling strength between the two mechanical modes as $g \equiv n_{\mathrm{ph}} \omega^{(2)} x_{\mathrm{ZPF}}^{2}$. Now, we come to the dynamics of the mechanical modes. From Heisenberg equation we get

$$
\begin{aligned}
\frac{d}{d t} b_{1}^{\dagger} b_{1} & =\frac{i}{\hbar}\left[H, b_{1}^{\dagger} b_{1}\right] \\
& =-i g b_{1}^{\dagger} b_{2}+i g b_{2}^{\dagger} b_{1} \\
\frac{d}{d t} b_{2}^{\dagger} b_{2} & =-i g b_{2}^{\dagger} b_{1}+i g b_{1}^{\dagger} b_{2}, \\
\frac{d}{d t} b_{1} & =-i \Omega_{\mathrm{m}} b_{1}-i g b_{2}, \\
\frac{d}{d t} b_{2} & =-i \Omega_{\mathrm{m}} b_{2}-i g b_{1} .
\end{aligned}
$$

The energy of the mechanical mode, $E=\hbar \Omega_{\mathrm{m}}\left\langle b_{i}^{\dagger} b_{i}\right\rangle$, with $i=1,2$, is described by the product of the eigenenergy of mode and the phonon number. Thus we can get the heat flow from the time derivative of the number operator $\frac{d}{d t} b_{i}^{\dagger} b_{i}$ in Eq. (6). Note that Eq. (6) ensures $\frac{d}{d t} b_{1}^{\dagger} b_{1}=$ $-\frac{d}{d t} b_{2}^{\dagger} b_{2}$ which is the conservation of the energy. As a result, we can focus on the equation of $\frac{d}{d t} b_{1}^{\dagger} b_{1}$ in the following derivation. The annihilation operators can be formally solved as

$$
\begin{aligned}
& b_{1}=e^{-i \Omega_{\mathrm{m}} t} b_{1}(0)-i g \int_{0}^{t} d s e^{-i \Omega_{\mathrm{m}}(t-s)} b_{2}(s), \\
& b_{2}=e^{-i \Omega_{\mathrm{m}} t} b_{2}(0)-i g \int_{0}^{t} d s e^{-i \Omega_{\mathrm{m}}(t-s)} b_{1}(s) .
\end{aligned}
$$

With the formal solution Eq. (7), the time derivative of the number operators can be expressed as

$$
\begin{aligned}
& \frac{d}{d t} b_{1}^{\dagger} b_{1}=-i g b_{1}^{\dagger}\left[e^{-i \Omega_{\mathrm{m}} t} b_{2}(0)-i g \int_{0}^{t} d s e^{-i \Omega_{\mathrm{m}}(t-s)} b_{1}(s)\right] \\
& +i g b_{2}^{\dagger}\left[e^{-i \Omega_{\mathrm{m}} t} b_{1}(0)-i g \int_{0}^{t} d s e^{-i \Omega_{\mathrm{m}}(t-s)} b_{2}(s)\right] \text {. }
\end{aligned}
$$

Then, we assume the coupling between the mechanical modes and the heat bathes to be strong, so that both mechanical oscillators can achieve thermal equilibrium with the bathes in a very short time. Thus we have

$$
\left\langle b_{i}^{\dagger}(t) b_{j}(s)\right\rangle=\delta_{i, j} \delta(t-s)^{-} n\left(T_{i}\right), \quad(i=1,2) .
$$

Here, $\bar{n}\left(T_{i}\right)=1 /\left(e^{\frac{\hbar \Omega_{\mathrm{m}}}{k_{\mathrm{B}} T_{i}}}-1\right)$ is the average phonon number at temperature $T$, the quanty $T_{i}$ describes the temperature of the bath coupled to mechanical mode $i$, and $\left\langle b_{i}^{\dagger}(t) b_{j}(s)\right\rangle$ is the expectation value of operator $b_{i}^{\dagger}(t) b_{j}(s)$. Then, the expectation value of Eq. (8) can be estimated,

$$
\frac{d}{d t}\left\langle b_{1}^{\dagger} b_{1}\right\rangle=-g^{2} \bar{n}\left(T_{1}\right)+g^{2} \bar{n}\left(T_{2}\right)
$$

Equation (9) provides the average number of the phonon that leaves the mechanical mode 1 per unit time. These phonons go to the mode 2 since we have shown $\frac{d}{d t} b_{1}^{\dagger} b_{1}=-\frac{d}{d t} b_{2}^{\dagger} b_{2}$. Note that the right side of Eq. (9) has an additional dimension of time, so that the both sides have the same dimension. This is the result of the relation $\int_{0}^{t} d s e^{-i \Omega_{\mathrm{m}}(t-s)}$ $\delta(t-s)^{-} n\left(T_{i}\right)=\bar{n}\left(T_{i}\right.$. The energy flux carried by phonon is

$$
\begin{aligned}
\Phi_{H} & =\hbar \Omega \mathrm{m} \frac{d}{d t}\left\langle b_{1}^{\dagger} b_{1}\right\rangle \\
& =\hbar \Omega \mathrm{m}\left[-g^{2} \bar{n}\left(T_{1}\right)+g^{2} \bar{n}\left(T_{2}\right)\right] .
\end{aligned}
$$

Where $\Delta T \equiv\left(T_{1}-T_{2}\right)$ is the temperature difference between the two bathes. Eq. (10) describes the rela-tion between the heat flux through the single device and the temperature difference in the two sides of the device. This relation has the same form as an ordinary object, but the coupling strength $g$ can be changed by the inten-sity of the pump light. If we turn off the light, the heat flux through the device can be shut down.

In the high temperature limit $k_{\mathrm{B}} T \gg \hbar \Omega_{\mathrm{m}}$, Eq. (10) becomes a simpler form

$$
\begin{aligned}
\Phi_{\mathrm{H}} & =-\hbar \Omega_{\mathrm{m}} g^{2}\left[\frac{\partial}{\partial T} \frac{1}{\left(e^{\frac{\hbar \Omega_{\mathrm{m}}}{k_{\mathrm{B}}}}-1\right)}\right] \Delta T \\
& \approx-\hbar \Omega_{\mathrm{m}} g^{2}\left[\frac{\partial}{\partial T} \frac{k_{\mathrm{B}} T}{\hbar \Omega_{\mathrm{m}}}\right] \Delta T \\
& =-k_{\mathrm{B}} g^{2} \Delta T .
\end{aligned}
$$

Regularly, thermal metamaterials work at room temperature. Thus, it always satisfies $k_{\mathrm{B}} T \gg \hbar \Omega_{\mathrm{m}}$. There-fore, in the rest of this article, we will always use the high-temperature limit. In a word, this simple optome-chanical system is the elemental unit of our adjustable thermal metamaterial just like the split-ring resonators (SRRs) adopted in the electro-magnetic cloak case.

\section{Thermal conductivity tuned by array of optomechanical systems}

We can arrange the optomechanical systems to form an array as shown in Fig. 2. Considering a two-dimensional array, the squares in the figure represent the heat bathes, which provide the local temperatures at different points. The thermal energy can conduct among those squares through the optomechanical systems connecting them.

Now, we are in a position to show that the new array system can work as controllable thermal metamaterial, and to derive the Fourier thermal conduction equation of it. Let's assume the distance between the nearby squares is $\mathrm{L}$ in both $\mathrm{X}$ and $\mathrm{Y}$ direction. We first consider the heat flux density in $\mathrm{X}$ direction. Suppose there is a line with length 1 in $\mathrm{Y}$ direction at coordinate $(\mathrm{x}, \mathrm{y})$, the number of the optomechanical systems that carry heat flux through it is $\frac{1}{L}$. If the heat flux carried by each optomechanical Lsystem is $\Phi_{x}$, then the flux density in X direction at that point should be

$$
J_{x}(x, y)=\frac{\Phi_{x}}{L}
$$

With Eq. (11), we can get

$$
J_{x}(x, y)=\frac{-k_{\mathrm{B}} g_{x}^{2}(x, y) \Delta T_{x}(x, y)}{L} .
$$

Here, $\Delta T_{x}(x, y)$ is the temperature difference between two closest bathes in $\mathrm{X}$ direction. The coupling strength $g_{x}(x, y)$ can be adjusted by the pump light on the op-tomechanical system at $(x, y)$ in $\mathrm{X}$ direction. We can approximate the quantity $\frac{\Delta T_{x}(x, y)}{L}$ with the derivative $\frac{\partial}{\partial x} T(x, y)$, so that the heat flux density in $\mathrm{X}$ direction can be expressed as

$$
J_{x}(x, y)=-k_{\mathrm{B}} g_{x}^{2}(x, y) \frac{\partial}{\partial x} T(x, y) .
$$


In the same way we can get the expression for the heat flux density in Y direction,

$$
J_{y}(x, y)=-k_{\text {в }} g_{y}^{2}(x, y) \frac{-}{\partial y} T(x, y) .
$$

With the heat flux density in both direction, the Fourier thermal conduction equation for the array can be obtained,

$$
\begin{aligned}
J(x, y) & =-\kappa(x, y) \nabla T(x, y), \\
\kappa_{x x}(x, y) & =k_{\mathrm{B}}\left[\omega^{(2)} x_{\mathrm{ZPF}}^{2} \frac{\mathcal{F}}{\hbar \omega \Delta \omega_{\mathrm{FSR}}}\right]^{2} P_{x}^{2}(x, y), \\
\kappa_{y y}(x, y) & =k_{\mathrm{B}}\left[\omega^{(2)} x_{\mathrm{ZPF}}^{2} \frac{\mathcal{F}}{\hbar \omega \Delta \omega_{\mathrm{FSR}}}\right]^{2} P_{y}^{2}(x, y), \\
\kappa_{x y}(x, y) & =\kappa_{y x}(x, y)=0 .
\end{aligned}
$$

where $P_{i}(x, y)$ is the pump power on the optomechanical system at $(x, y)$ in I direction $i=x, y$. We can find from Eq. (12) that the array of optomechanical systems has the thermal conductivity $\kappa(x, y)$ related to the square of the pump power. If we choose an uniform pump power $P_{x}(x, y)=P_{y}(x, y)=P_{0}$ in some area, this array has the same property as a ordinary material. A large variety of thermal metamaterails can be made by combination of different ordinary materials. ${ }^{34}$ For our array, we just need to adjust the pump power in different position. Thus the array can be switched among different kinds of metamaterials with all kinds of function.

Now, we discuss the necessary pump power for this array. For a given pump power, the thermal conductivity is decided by the coeffcient

$k_{\mathrm{B}}\left[\frac{\omega^{(2)} x_{\mathrm{ZPF}}^{2} \mathcal{F}}{\hbar \omega \Delta \omega_{\mathrm{FSR}}}\right]^{2}$, where $k_{\mathrm{B}}$ and $\hbar$ are constants which can not be changed. Other parameters, which are related to the properties of optomechanical systems, can be divided into three parts. The first part $\frac{\omega^{(2)}}{\omega}$ is related to the strength of the second-order coupling between the optical and mechanical modes. To enhance this term a large $\omega^{(2)}=\frac{\partial^{2}}{\partial x_{1} \partial x_{2}} \omega$ and a small $\omega$ are necessary. We expect the $\omega^{(2)}$ will have a similar magnitude to the one of $\omega^{\prime \prime} \equiv \frac{\partial^{2}}{\partial x^{2}} \omega$ in "membrane in middle" optomechanical system which is about $10 \mathrm{MHz} \mathrm{nm}$. The frequency of the light mode is about $10^{14} \mathrm{~Hz} .^{31}$ Thus the $\omega^{(2)}$ can reach the mag-nitude of $10^{-7} \mathrm{~nm}^{-2}$ or $10^{11} \mathrm{~m}^{-2}$. The next part $x_{\mathrm{ZPF}}$ is decided by the effective mass and frequency of the mechanical oscillator. Optomechanical systems formed by nanoscale oscillator ${ }^{32}$ or clod atom ${ }^{33}$ usually have large $x_{\mathrm{ZPF}}$. Here, we take the parameter of the nanoscale oscillator in our estimation, which has the frequency in the order of $10^{9} \mathrm{~Hz}$ and the effective mass in the order of $10^{-16} \mathrm{~kg}$. Final part $\frac{\mathcal{F}}{\Delta \omega_{\mathrm{FSR}}}$ represents the leakage of the cavity. For optomechanical systems, this term can be about $10^{-5} \mathrm{~S} .{ }^{27}$ With the parameters mentioned above, we can estimate the necessary pump power on an individual optomechanical unit for a certain thermal conductivity.

Here, we estimate the upper bond of the pump power used in the array system to achieve the usual materials' conductivities. Consider the thermal flux $\Phi_{H}$ through a cuboid with a thickness of $H$, a width of $D$ and a length of $L$. This flux can be calculated by

$$
\Phi_{H}=\frac{\kappa D H}{L}\left(T_{1}-T_{2}\right)
$$

with $\kappa$ the thermal conductivity of the material compris-ing the cuboid. Then, we view the cuboid from the above and treat it as a twodimension object. The effective two-dimension thermal conductivity of the correspond-ing "material" is

$$
\kappa_{2 D}=\frac{\Phi_{H} L}{D\left(T_{1}-T_{2}\right)}=\kappa H .
$$

Therefore, the effective thermal conductivity in a two-dimension setup is just the thermal conductivity of the material multiplied by the thickness. If the thickness of the structure is homogenous, the effective two-dimensional thermal conductivity of the material can be directly used in designing the metamaterial. We take the copper slice, which holds the highest thermal conductivity among the common materials one can easily obtain from nature, as an example. The thermal conductivity of copper is about $4 \times 10^{2} \mathrm{~W} \mathrm{~m}^{-1} \mathrm{~K}^{-1}$, and assume the thickness of the copper slice is $0.1 \mathrm{~mm}$. Thus the two-dimensional thermal conductivity of the copper slice is $\times 10^{-2} \mathrm{~W} \mathrm{~K}^{-1}$. To achieve the thermal conduction effect of a copper slice, the necessary pump power is about $10^{-2} \mathrm{~W}$. Such a pump is very strong compared with the usual

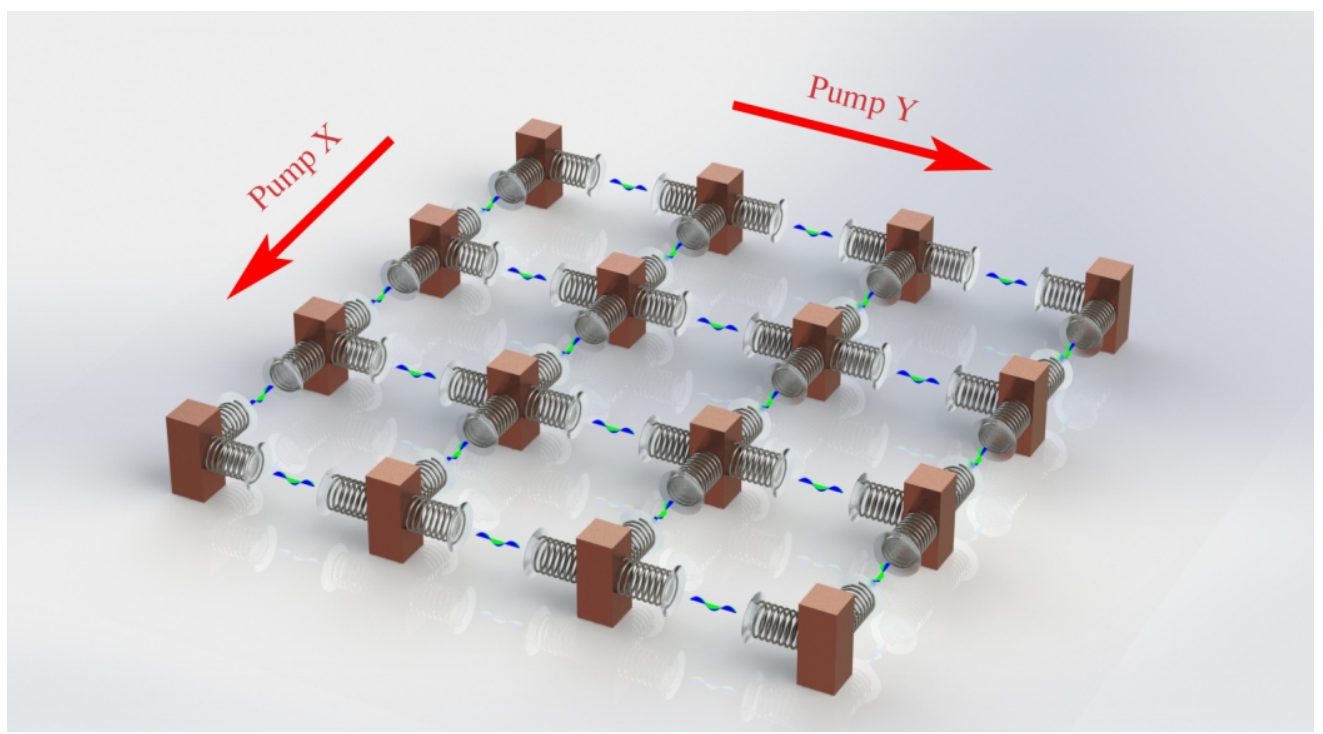

Fig. 2 Blueprint of the array formed by optomechanical systems. The cubes made of ordinary material are connected by the optomechanical systems. The array is pumped by inhomogeneous light field. 
pump in optomechanical systems, but note that we do not need any coherence or quantum properties in our design. Therefore, the photon noise, which constrains the pump in quantum optomechanical systems, is not a problem here. Also, the pump is usually largely detuned from the oscillator, so that the heating effect of the pump light is weak.

\section{Design of thermal meta-device by utilizing optomechanical array system}

In this section we give the example of how to design multiple thermal metadevices by using the aforementioned array system of optomechanical resonators. For demonstration, we adopt the simplified design of bilayer thermal cloak and concentrator with reduced thermal parameters. ${ }^{13,19}$ The bilayer cloak has two parts as shown in the Fig. 3 (a): the thermal insulator inner ring and a outer ring with a specific thermal conductivity. Denote the background $(\mathrm{r}>\mathrm{c})$, the outer ring $(\mathrm{c}<\mathrm{r}<\mathrm{b})$, the inner ring $(\mathrm{b}<\mathrm{r}<$ a) and the invisible area $(\mathrm{r}<\mathrm{a})$ to be region 1, 2, 3, 4 respectively. As indicated in ref [13], for a two dimensional system, if the thermal conductivity of the background is $\kappa_{\mathrm{b}}$ then the thermal conductivity distribution of the cloak can be derived as:

$$
\left\{\begin{array}{l}
\kappa_{1}^{\text {cloak }}=\kappa_{b} \\
\kappa_{2}^{\text {cloak }}=\kappa_{b} \frac{\left(c^{2}+b^{2}\right)}{\left(c^{2}-b^{2}\right)} \\
\kappa_{3}^{\text {cloak }}=0 \\
\kappa_{4}^{\text {cloak }}=C \kappa_{b}
\end{array}\right.
$$

(a)

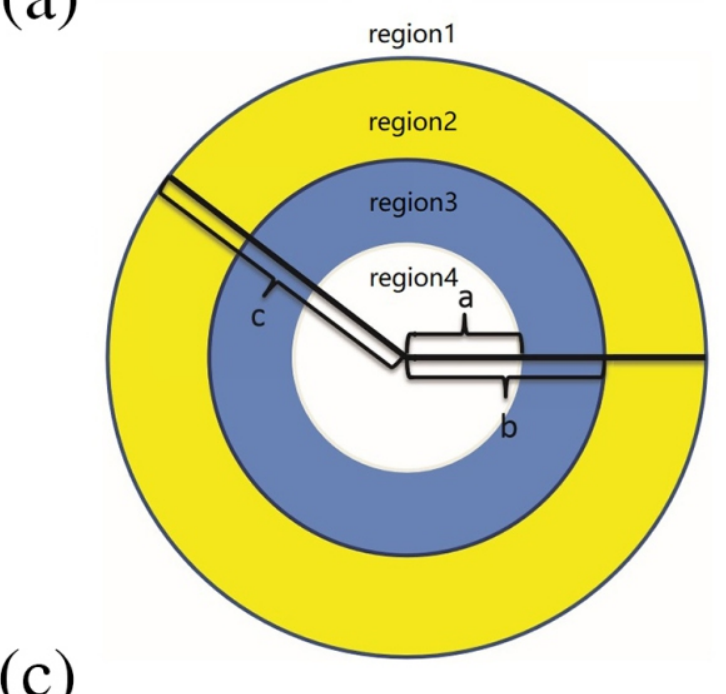

(b)

(d)

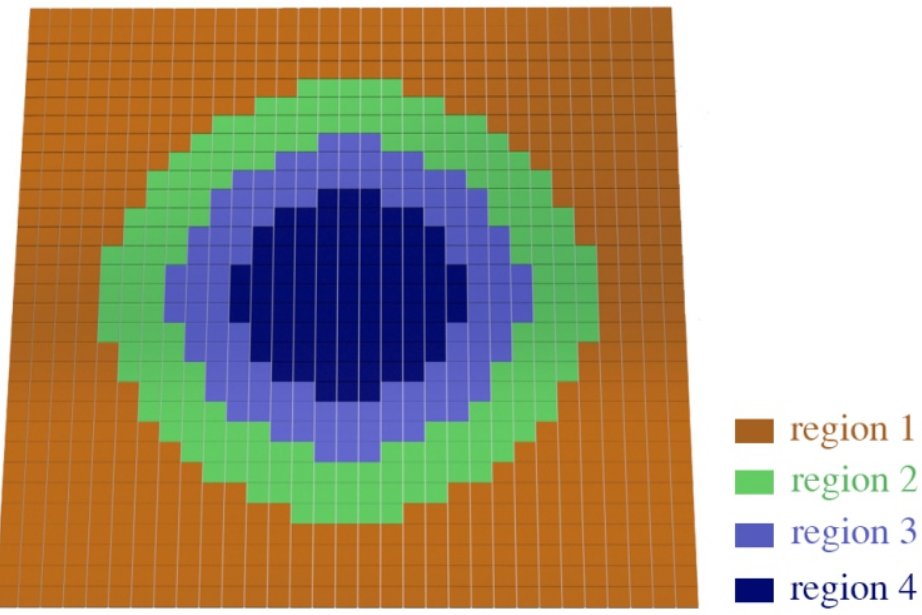

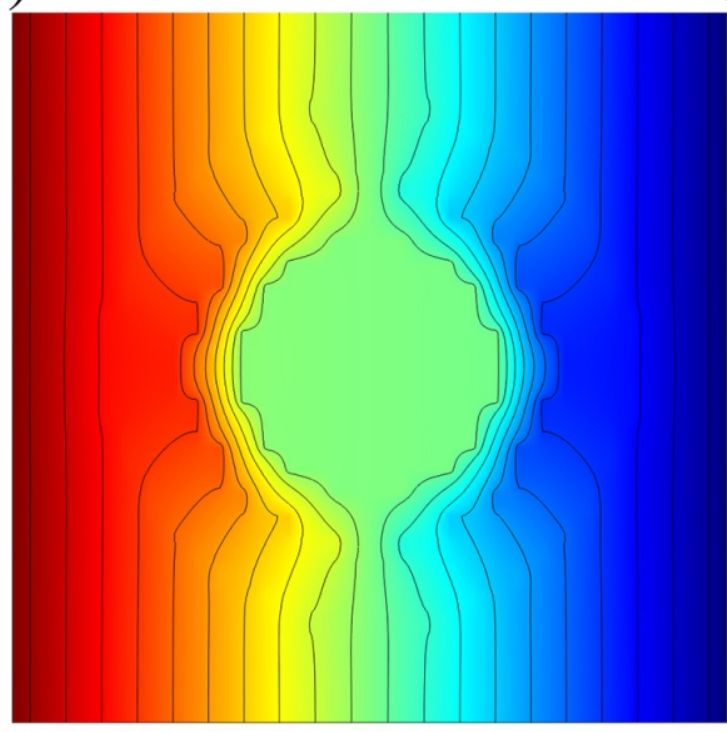

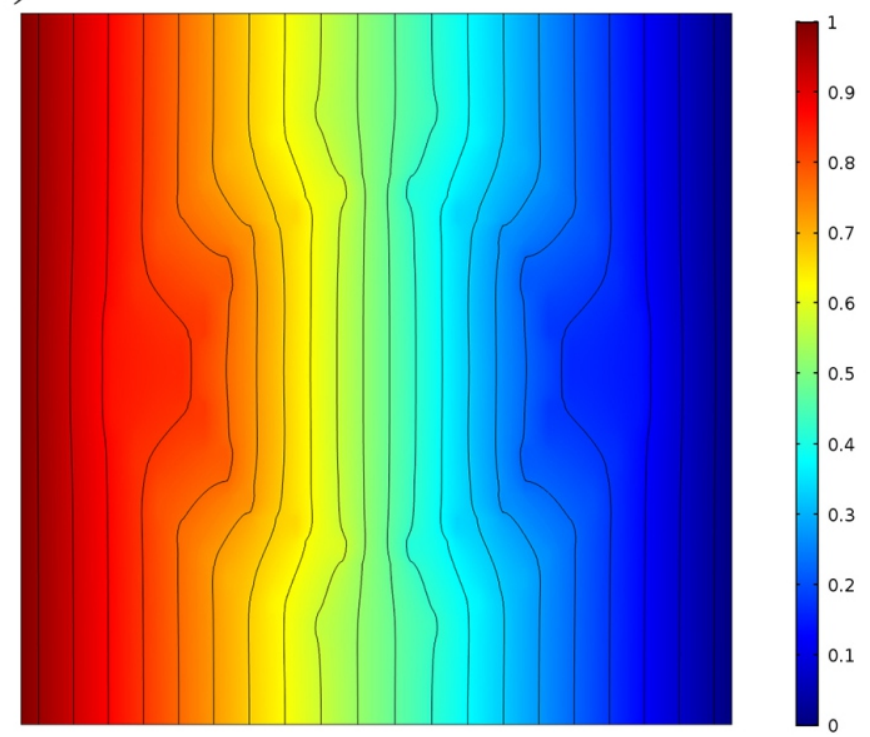

Fig. 3 (a) The figure shows the bilayer thermal cloak or concentrator. The region 1 is the background, in which $r>c$. The region 2 and 3 are the outer ring $(\mathrm{c}<\mathrm{r}<\mathrm{b})$ and the inner ring $(\mathrm{b}<\mathrm{r}<\mathrm{a})$ respectively. The region 4 is concealed in the center of the device within the $\mathrm{r}<\mathrm{a}$ domain, and represents the invisible or concentrating area. (b) The blueprint of our thermal device design by utilizing the array of optomechanical systems. There are $31 \times 31$ unit cells indicated by the small squares in the device, and for the di erent regions denoted by di erent colors the thermal conductivities can be tuned according to the cloaking or concentrating parameters. (c) The simulation result shows the snapshot of the temperature distribution for a thermal cloak device. (d) The temperature profile for a thermal concentrator derived from the simulation is also given. 
where $C$ is an arbitrary constant representing the conductivity of the object hidden inside the cloak. On the other hand, a thermal concentrator can also be achieved by using this bilayer structure, ${ }^{19}$ and the corresponding parameters are given below:

$$
\left\{\begin{array}{l}
\kappa_{1}^{\text {con }}=\kappa_{b} \\
\kappa_{2}^{c o n}=\kappa_{b} \frac{\left(c^{2}+b^{2}\right)}{\left(c^{2}-b^{2}\right)} \\
\kappa_{3}^{c o n}=D \kappa_{b} \\
\kappa_{4}^{c o n}=\kappa_{3}^{c o n} \frac{b^{2}-a^{2} \kappa_{3}^{c o n}}{a b+a^{2}}
\end{array}\right.
$$

here $D \kappa_{b}$ is a smaller conductivity compared to the $\kappa_{b}\left(c^{2}+b^{2}\right) /\left(c^{2}-b^{2}\right)$ playing a role to eliminate the distortion of the temperature distribution outside the device.

To achieve the function of the bilayer thermal cloak or concentrator, we just need to set the pump power according to the original design and the Eq. (12). Let's assume the center of the thermal bilayer cloak locates at $\left(x_{0}, y_{0}\right)$ and the pump power of the background (the area with $\left.\left(x-x_{0}\right)^{2}+\left(y-y_{0}\right)^{2}>c^{2}\right)$ is $P_{0}$. To make the inner ring of the cloak thermal insulator, we can simply shut down the pump. Therefore, we obtain the pump power for the different region to achieve the cloaking effect:

$$
\left\{\begin{array}{l}
P_{x}(x, y)=P_{y}(x, y)=P_{0}, \text { for } r>c \\
P_{x}(x, y)=P_{y}(x, y)=\sqrt{\frac{c^{2}+b^{2}}{c^{2}-b^{2}}} P_{0}, \text { for } c>r>b \\
P_{x}(x, y)=P_{y}(x, y)=0, \text { for } b r>a \\
P_{x}(x, y)=P_{y}(x, y)=\sqrt{C} P_{0}, \text { for } r<a
\end{array}\right.
$$

On the same footing, the pump power distribution for the concentrator can be calculated as

$$
\left\{\begin{array}{l}
P_{x}(x, y)=P_{y}(x, y)=P_{0}, \text { for } r>c \\
P_{x}(x, y)=P_{y}(x, y)=\sqrt{\frac{c^{2}+b^{2}}{c^{2}-b^{2}}} P_{0}, \text { for } c>r>b \\
P_{x}(x, y)=P_{y}(x, y)=\sqrt{D P_{0}, \text { for } b>r>a} \\
P_{x}(x, y)=P_{y}(x, y)=\sqrt{D \frac{b^{2}-a^{2} D}{a b+a^{2}}} P_{0}, \text { for } r<a
\end{array}\right.
$$

Now, we are in a position to give a more practical de-sign for the array system. Illustrated in Fig. 3(b), just as a picture consisting of pixels, we construct the array net-work with $31 \times 31$ pixels and each pixel indicates a unit cell of the optomechanical resonator. The regions 1, 2, 3, 4 are denoted by the different colors, and the necessary pump powers for these regions can be calculated by approximating the green and blue regions as the annuluses. Hence, for a cloak the pump powers are given:

$$
\left\{\begin{array}{l}
P_{x}(x, y)=P_{y}(x, y)=P_{0}, \text { for } r>c \\
P_{x}(x, y)=P_{y}(x, y)=1.852 P_{0}, \text { for } c>r>b \\
P_{x}(x, y)=P_{y}(x, y)=0, \text { for } b>r>a \\
P_{x}(x, y)=P_{y}(x, y)=\text { arbitrary value, for } r<a
\end{array}\right.
$$

For the concentrator, the parameters are:

$$
\left\{\begin{array}{l}
P_{x}(x, y)=P_{y}(x, y)=P_{0}, \text { for } r>c \\
P_{x}(x, y)=P_{y}(x, y)=1.852 P_{0}, \text { for } c>r>b \\
P_{x}(x, y)=P_{y}(x, y)=0.316 P_{0}, \text { for } b>r>a \\
P_{x}(x, y)=P_{y}(x, y)=0.3 P_{0}, \text { for } r<a
\end{array}\right.
$$

Taking account of the above two sets of pump power val-ues, we perform the simulations to investigate the thermal conduction behaviors of this array placed in a temperature field with a normalized temperature gradient. The simulation results are shown in the Figs. 3C) (d), and the temperature profiles are in good agreement with the anticipations. Apparently, under such a pump dis-tribution the array works as a bilayer cloak or a concentrator. It needs to be emphasized that these two thermal metamaterials are just adopted as the demonstration samples. In fact, almost all the existing thermal metadevices can be achieved by such an array network. As aforementioned, if we change the pump power, the thermal conductivity of the related region thus will be changed. Hence, when we tune the thermal conductivity of the outer and inner ring to the same value as the background, we cancel the cloaking function of the system. Besides, if we tune the pump power of region 3 and region 4 , the device may switch from a cloak to a concentrator or vice versa. Obviously, for this new adjustable array system, by providing different pump power, switchable multi-functional thermal metamaterials can be easily realized.

\section{Conclusion}

In this article, we propose a new method to fabricate programmable thermal metamaterial by utilizing the optomechanical system as an elemental unit. After theoretical analysis, we find that the thermal conductivity of this unit is proportional to the square of the pump light intensity, and therefore can be tuned by providing different pump power. For instance, we also show how to build a switchable thermal metamaterial by adopting an optomechanical heat-conducting unit. Moreover, for experimental instructions, the necessary pump power is given as a reference. The new method provides more freedom and flexibility to assemble existing functions of thermal metamaterials to only one device, whose conductivities have quick responses to the pump light and can be adjusted according to the different demands. The array system consists of optomechanical units that enable the design of multi-functions materials that can be easily programmed with functionshifting tasks, which means that the material can be switched among any types of known thermal devices (cloak, concentrator, rotator, inverter and so on). We anticipate this approach can be extended to various directions, and help people designing more sorts of metamaterials with fantasy characteristics.

\section{Acknowledgments}

We thank Dr. Ying Li for helpful assistance and discus-sion. We acknowledge the financial support by the National Natural Science Foundation of China under Grant No. 11725521

\section{References}

1. C. Z. Fan, Y. Gao and J. P. Huang, Appl. Phys. Lett., 2008, 92, 251907.

2. S. Narayana and Y. Sato, Phys. Rev. Lett., 2012, 108, 214303.

3. L. J. Xu, S. Yang and J. P. Huang, Phys. Rev. Appl., 2019, 11, 054071.

4. L. J. Xu and J. P. Huang, EPL, 2019, 125, 64001.

5. L. J. Xu, S. Yang and J. P. Huang, Phys. Rev. E, 2019, 99, 022107.

6. H. Y. Xu, X. H. Shi, F. Gao, H. D. Sun and B. L. Zhang, Phys. Rev. Lett., 2014, 112, 054301.

7. Y. Gao and J. P. Huang, EPL, 2013, 104, 44001.

8. X. Y. Shen and J. P. Huang, Int. J. Heat Mass Transfer, 2014, 78, 1.

9. T. C. Han, T. Yuan, B. W. Li and W. C. Qiu, Sci. Rep., 2013, 3, 1593.

10. T. C. Han, J. Zhao, T. Yuan, D. Y. Lei, B. W. Li and C. W. Qiu, Energy Environ. Sci., 2013, 6, 3537.

11. T. C. Han, X. Bai, J. T. L. Thong, B.W. Li and C. W. Qiu, Adv. Mat., 2014, 26, 1731 
12. R. Schittny, M. Kadic, S. Guenneau and M. Wegener, Phys. Rev. Lett., 2013, 110, 195901.

13. T. C. Han, X. Bai, D. L. Gao, J. T. L. Thong, B. W. Li and C. W. Qiu, Phys. Rev. Lett., 2014, 112, 054302.

14. Y. Ma, Y. Liu, M. Raza, Y. Wang and S. He, Phys. Rev. Lett., 2014, 113, 205501.

15. J. Y. Li, Y. Gao and J. P. Huang, J. Appl. Phys., 2010, 108, 074504.

16. T. Z. Yang, X. Bai, D. L. Gao, L. Z. Wu, B. W. Li, J. T. L. Thong and C. W. Qiu, Adv. Mater, 2015, 27, 7752.

17. Y. Li, X. Y. Shen, Z. H. Wu, J. Y. Huang, Y. X. Chen, Y. S. Ni and J. P. Huang, Phys. Rev. Lett., 2015, 115, 195503.

18. X. Y. Shen, Y. Li, C. R. Jiang and J. P. Huang, Phys. Rev. Lett., 2016, 117, 055501.

19. X. Y. Shen, Y. Li, C. R. Jiang, Y. S. Ni and J. P. Huang, Appl. Phys. Lett., 2016, 109, 031907.

20. Y. Li, K. J. Zhu, Y. G. Peng, W. Li, T. Yang, H. X. Xu, H. Chen, X. F. Zhu, S. Fan and C. W. Qiu, Nat. Mat., 2019, 18, 48.

21. Y. Li, Y. G. Peng, L. Han, M. A. Miri, W. Li, M. Xiao, X. F. Zhu, J. Zhao, A. Al'u, S. Fan and C. W. Qiu, Science, 2019, 364, 170.

22. H. Zhang, K. Hippalgaonkar, T. Buonassisi, O. M. Løvvik, E. Sagvolden and D. Ding, ES Energy Environ., 2018, 2, 1-8.

23. M. E. Navarro, A. Ahmad, Y. M. Luo and X. H. She, ES Energy Environ., $2019,4,1-4$
24. C. Coulais, E. Teomy, K. Reus, Y. Shokef and M. Hecke, Nature, 2016, 535, 529 .

25. A. Balandin and K. L. Wang, Phys. Rev. B, 1998, 58, 1544.

26. B. L. Davis and M. I. Hussein, Phys. Rev. Lett., 2014, 112, 055505.

27. M. Aspelmeyer, T. J. Kippenberg and F. Marquardt, Rev. Mod. Phys., 2014, 86, 1391.

28. M. Zalalutdinov, A. Zehnder, A. Olkhovets, S. Turner, L. Sekaric, B. Llic, D. Czaplewski, J. M. Parpia and H. G. Craighead, Appl. Phys. Lett., 2001, 79, 695.

29. P. F. Cohadon, A. Heidmann and M. Pinard, Phys. Rev. Lett., 1999, 83, 3174.

30. M. Vogel, C. Mooser, K. Karrai and R. J. Warburton, Appl. Phys. Lett., 2003, 83, 1337.

31. J. C. Sankey, C. Yang, B. M. Zwickl, A. M. Jayich and J. G. E. Harris, Nat Phys., 2010, 6, 707.

32. J. Chan, T. P. M. Alegre, A. H. Safavi-Naeini, J. T. Hill, A. Krause, S. Gr"ublacher, M. Aspelmeyer and O. Painter, Nature, 2011, 478, 89.

33. K. W. Murch, K. L. Moore, S. Gupta and D. M. Stamper-Kurn, Nat. Phys., 2008, 4, 561.

34. M. Kadic, T. B"uckmann, R. Schittny and M. Wegener, Pep. Prog. Phys., 2013, 76, 126501.

Publisher's Note Engineered Science Publisher remains neutral with regard to jurisdictional claims in published maps and institutional affiliations. 